\title{
Intestinal clock system regulates skeletal homeostasis
}

\author{
Masanobu Kawai, ${ }^{1}$ Saori Kinoshita, ${ }^{1}$ Miwa Yamazaki, ${ }^{1}$ Keiko Yamamoto, ${ }^{1}$ Clifford J. Rosen, ${ }^{2}$ \\ Shigeki Shimba, ${ }^{3}$ Keiichi Ozono, ${ }^{4}$ and Toshimi Michigami' \\ 'Department of Bone and Mineral Research, Research Institute, Osaka Women's and Children's Hospital, Izumi, Osaka, \\ Japan. ${ }^{2}$ Maine Medical Center Research Institute, Scarborough, Maine, USA. ${ }^{3}$ Department of Health Science, School of \\ Pharmacy, Nihon University, Funabashi, Chiba, Japan. ${ }^{4}$ Department of Pediatrics, Osaka University Graduate School of \\ Medicine, Suita, Osaka, Japan.
}

The circadian clock network is an evolutionarily conserved system involved in the regulation of metabolic homeostasis; however, its impacts on skeletal metabolism remain largely unknown. We herein demonstrated that the circadian clock network in the intestines plays pivotal roles in skeletal metabolism such that the lack of the Bmal1 gene in the intestines ( $B \mathrm{mal1}{ }_{\mathrm{Int}}^{-1-}$ mice) caused bone loss, with bone resorption being activated and bone formation suppressed. Mechanistically, Clock protein interaction with the vitamin D receptor (VDR) accelerated its binding to the VDR response element by enhancing histone acetylation in a circadian-dependent manner, and this was lost in Bmal1 $_{\text {Int }}{ }^{-1-}$ mice because nuclear translocation of Clock required the presence of Bmal1. Accordingly, the rhythmic expression of VDR target genes involved in transcellular calcium (Ca) absorption was created, and this was not observed in $\mathrm{Bmal1}_{\mathrm{Int}}{ }^{-/-}$mice. As a result, transcellular $\mathrm{Ca}$ absorption was impaired and bone resorption was activated in Bmal1 ${ }_{\mathrm{Int}}{ }^{-/-}$mice. Additionally, sympathetic tone, the activation of which suppresses bone formation, was elevated through afferent vagal nerves in $\mathrm{Bmal1}_{{ }_{\mathrm{Int}}}^{-1-}$ mice, the blockade of which partially recovered bone loss by increasing bone formation and suppressing bone resorption in Bmal1 ${ }_{\text {Int }}{ }^{-1-}$ mice. These results demonstrate that the intestinal circadian system regulates skeletal bone homeostasis.

Conflict of interest: The authors have declared that no conflict of interest exists.

License: Copyright 2019, American Society for Clinical Investigation.

Submitted: May 8, 2018

Accepted: January 30, 2019

Published: March 7, 2019

Reference information: JCI Insight. 2019;4(5):e121798. https://doi.org/10.1172/jici. insight.121798.

\section{Introduction}

The circadian clock network is an evolutionarily conserved system by which organisms adapt their metabolic activities to environmental inputs including the availability of nutrients. The master pacemaker of the circadian clock system is located in the suprachiasmatic nucleus (SCN) (1) and is well known to play pivotal roles in metabolic regulation; therefore, the disruption of this system has been linked to the development of metabolic complications such as obesity and glucose intolerance (2-4). In addition to the central pacemaker, peripheral tissues possess their own circadian system and synchronize with the central clock system through hormonal and neuronal signals (2). Importantly, the peripheral circadian system is entrained by external cues such as food availability independent of central regulation, and indeed time-restricted feeding during the light phase has been shown to reverse the expression profiles of core clock genes without affecting their cycling in the SCN, indicating that the feeding/fasting cycle drives peripheral circadian rhythmicity (5). Organisms take advantage of this system to allow for the predictable time-of-day-dependent utilization of ingested nutrients by optimizing the metabolic processes of nutrients in peripheral tissues.

Intestinal tissue is a critical organ in the regulation of nutrient metabolism and is well known to be governed by the circadian clock system. As is the case with other metabolic organs such as the liver, time of feeding also regulates the expression profile of clock genes in the intestines $(6,7)$. Consistent with this notion, multiple intestinal activities such as nutrient absorption are regulated by the circadian clock system (8-10). Calcium $(\mathrm{Ca})$ is one of the important nutrients actively and transcellularly absorbed mainly from the duodenum (11) and plays pivotal roles in skeletal metabolism. Ca homeostasis is regulated in a manner involving the circadian network because a circadian profile has been reported for transcellular $\mathrm{Ca}$ absorption (12) and serum Ca levels (13); however, its underlying molecular mechanisms have not been fully understood. 
Several lines of evidence demonstrate that the disturbance of the circadian clock system affects skeletal metabolism (14-17). Although mice globally lacking the Bmall gene have been shown to exhibit decreased bone mass, which was in part explained by its deletion in osteoblastic cells (14), the lack of the Bmall gene in osteoclastic cells enhanced bone mass by suppressing osteoclast differentiation and activity in mice (16), indicating that the effects of the peripheral clock on bone metabolism are site and context specific, and the exact role of the molecular clock network on bone metabolism still needs to be clarified. Based on the accumulating evidence of a critical role for the intestine as a nodal organ integrating inter-organ communication through nutritional, humoral, and neuronal pathways, we hypothesized that the disruption of the circadian clock system in the intestines impairs $\mathrm{Ca}$ absorption and causes deleterious effects on skeletal metabolism; however, to our knowledge this scenario has not been tested so far.

Active Ca transport is exerted in a manner involving the vitamin D receptor (VDR) signaling pathway $(18,19)$, activation of which has been shown to enhance the expression of genes involved in transcellular Ca absorption including TRPV6, CABP9k (coding for calbindin-D9k), and ATP2B1 (coding for Pmca1). The importance of the VDR in transcellular Ca absorption was evidenced by the fact that the lack of VDR in the intestines decreased $\mathrm{Ca}$ absorption (20). Importantly, circulating $\mathrm{Ca}$ levels were maintained in part by driving Ca mobilization from the bone in these mice, which resulted in decreased bone mass (20). Since VDR expression has been suggested to be rhythmic in a given tissue (21), we specifically hypothesized that the circadian clock system in the intestine regulates VDR activity and the alterations in the circadian clock network in the intestine affect bone metabolism by disrupting $\mathrm{Ca}$ homeostasis.

In order to test this hypothesis, we utilized a mouse model in which the Bmal1 gene was conditionally deleted in the intestines, and found that Clock (circadian locomotor output cycles kaput) physically and functionally interacted with VDR and created rhythmicity in the expression of VDR target genes, which resulted in impaired transcellular $\mathrm{Ca}$ absorption and caused compensatory activation of bone resorption. Furthermore, we found that the lack of Bmal1 in the intestines suppressed bone formation and activated bone resorption through neuronal circuits, which included activation of sympathetic tone through afferent vagal nerves. As a result, the disruption of the clock network in the intestines reduced bone mass.

\section{Results}

Generation of Bmal1 ${ }_{\text {Int }}^{-1-}$ mice. In order to elucidate the skeletal consequences of disrupted biological rhythms in the intestines, we generated mice lacking the Bmall gene in the intestines by crossing Bmal1 ${ }^{f / f l}$ mice with Villin-Cre mice (Bmal1 Int $^{-1-}$ mice) (Supplemental Figure 1A; supplemental material available online with this article; https://doi.org/10.1172/jci.insight.121798DS1). The excision of Bmal1 in the villi of the duodenum was confirmed, as shown in Supplemental Figure 1, B-D. No significant deletion was noted in extra-intestinal tissues including the hypothalamus (Supplemental Figure 1E). The expression of genes involved in the circadian clock network was disrupted in the villi of the duodenum obtained from Bmall $_{\text {Int }^{-1}}$ mice (Figure 1A). Bmal1 ${ }_{\text {Int }}^{-/-}$mice did not show any significant differences in body weight, tail length, food and water intake, locomotor activity, or wheel-running activity records under LD (12-hour light/12-hour dark) or DD (constant darkness) cycles from the controls, suggesting that the central clock network is unlikely affected in Bmal1 $_{\text {Int }}^{-/-}$mice (Figure 1B, and Supplemental Figure 2, A-F). Histological analysis of the duodenum showed no significant changes between the 2 groups (Supplemental Figure 2G).

Circadian expression profiles of VDR target genes in the intestines is disrupted in Bmal1 ${ }_{\text {Int }}^{-1-}$ mice. In the present study, we utilized male mice because $\mathrm{Ca}$ absorption in female mice is affected by the reproductive cycle (22). The expression profiles of genes involved in transcellular $\mathrm{Ca}$ absorption in the villi of the duodenum, including Trpv6, Cabp9k, and Atp2b1, showed a rhythmic expression pattern with a peak at ZT8 (zeitgeber time: light phase, ZT0-ZT12; dark phase, ZT12-ZT24) in control mice; this peak shifted to ZT12, with a weaker amplitude in Bmal1 ${ }_{\text {Int }}^{-1-}$ mice (Figure 1C). Because these are VDR target genes, we speculated that the circadian recruitment of VDR to the VDR response element (VDRE) created rhythmicity in VDR target genes. A ChIP analysis performed 1 and 4 hours after 1,25-(OH $)_{2} \mathrm{D}_{3}$ injection confirmed that the recruitment of VDR to the VDRE showed a rhythmic pattern that was stronger at ZT8 in control mice, and this disappeared in Bmall $_{\text {Int }}{ }^{-/-}$mice (Figure 1D).

VDR expression displays a rhythmic pattern. The expression of $V d r$ and VDR also peaked at ZT8 in control mice, and this disappeared in Bmal1 ${ }_{\text {Int }}^{-1-}$ mice (Figure 2, A and B). Furthermore, an in vitro analysis using Caco2 cells revealed that the knockdown of BMAL1 decreased VDR and VDR expression (Supplemental Figure 3, A-C). These results prompted us to speculate that the Bmall/Clock complex regulates the expression of $V d r$ through enhancer boxes (E-boxes) located in the $V d r$ gene locus. Although an in silico 
A
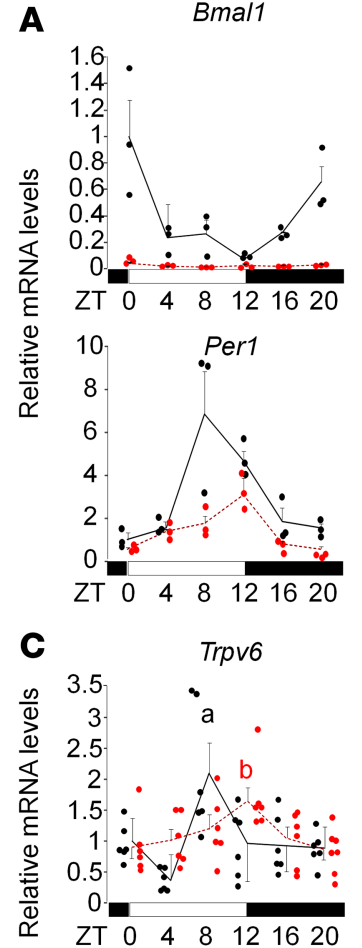
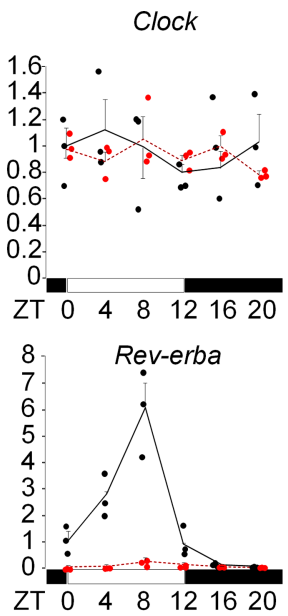

Cabp9k

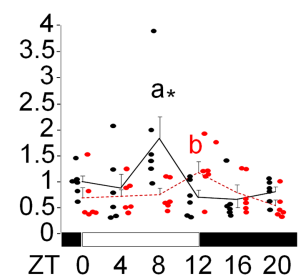

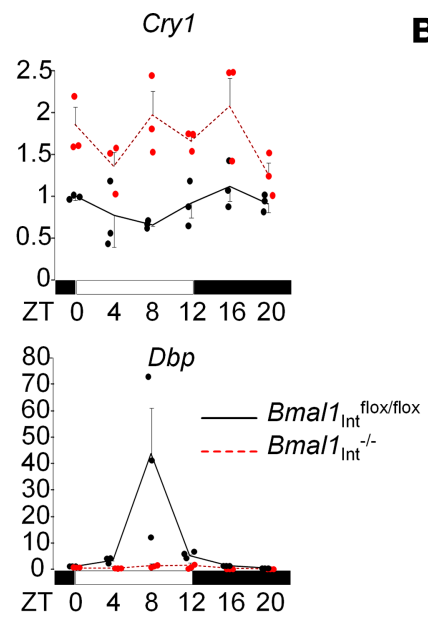

B

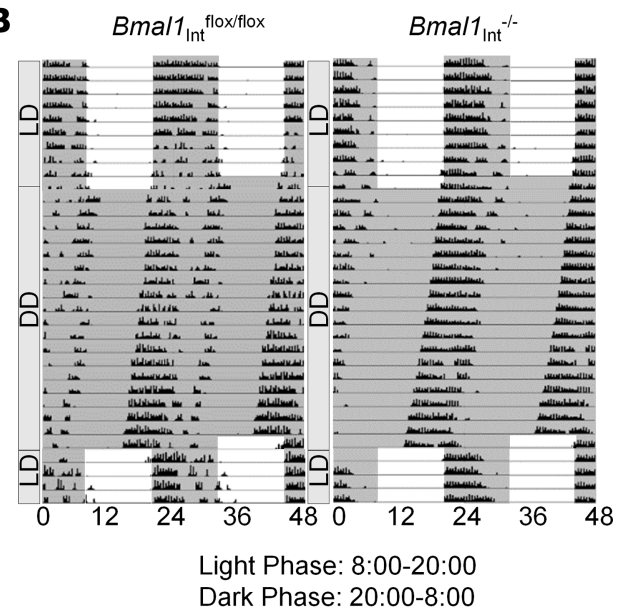

Pmca1b

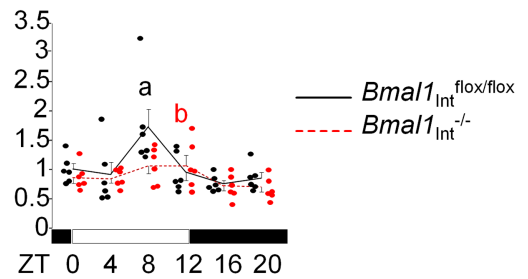

D

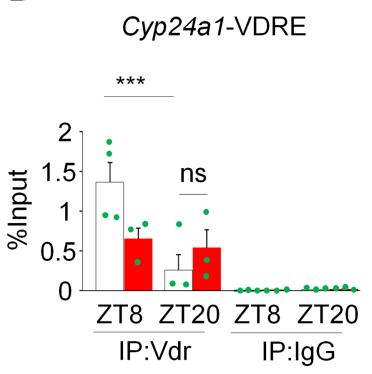

1-hour post VD injection

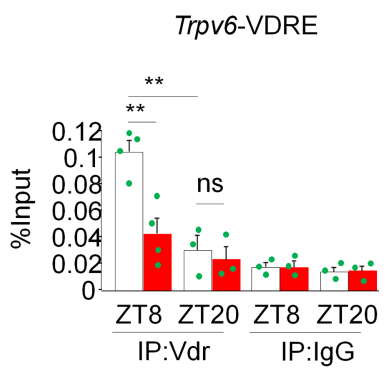

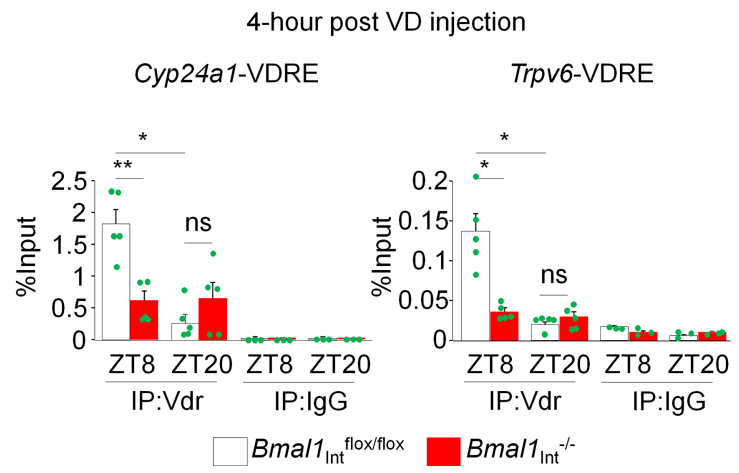

Figure 1. Rhythmic recruitment of VDR at the VDR target genes disappears in Bmal1 ${ }_{{ }_{\text {ntt }}}^{-1-}$ mice. (A) Villi were collected from the duodenum at 8 weeks of age every 4 hours, and expression of clock genes of interest was determined by real-time RT-PCR $(n=3)$. (B) Wheel-running activity was recorded and

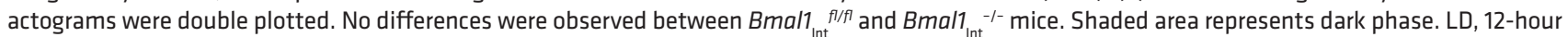
light/12-hour dark cycle; DD, constant darkness. A representative of at least 3 independent experiments is shown. (C) Villi were collected from the duodenum at 8 weeks of age every 4 hours, and expression of genes involved in transcellular Ca transport was determined by real-time RT-PCR ( $n=6)$. Trpv6 (a): $P<0.05$, ZT8 vs. ZTO; $P<0.01$, ZT8 vs. ZT12 and ZT16; $P<0.001$, ZT8 vs. ZT4 and ZT20; in Bmal1 fl/fl mice, by 1-way ANOVA. Trpv6 (b): $P<0.05, Z T 12$ vs. ZTO, ZT4, and ZT16; $P<0.01$, ZT12 vs. ZT20; in Bmal1 ${ }_{\text {Int }}^{-1-}$ mice, by 1-way ANOVA. Cabp9k (a): $P<0.05$, ZT8 vs. ZTO; $P<0.01, Z$ T 8 vs. ZT4, ZT12, ZT16, and ZT20; in Bmal1 ${ }_{\text {Int }}^{\text {flfl }}$ mice, by 1-way ANOVA. Cabp9k (b): $P<0.05$, ZT12 vs. ZT0 and ZT4; $P<0.01$, ZT12 vs. ZT20; in Bmal1 ${ }_{\text {Int }}^{-1-}$ mice, by 1-way ANOVA. Pmca1b (a): $P<0.05$, ZT8 vs. ZT20; $P<0.01$, ZT8 vs. ZTO, ZT4 and ZT12; $P<0.001$, ZT8 vs. ZT20; in Bmal1 fl/fl mice, by 1-way ANOVA. $P$ mca1b (b): $P<0.05, Z T 12$ vs.

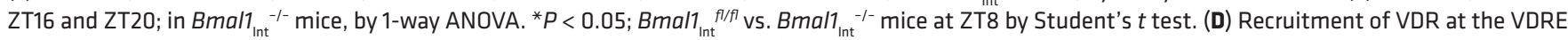
of Cyp24a1 and Trpv6 genes was analyzed 1 and 4 hours after 1,25-(OH) $\mathrm{D}_{3}(\mathrm{VD})$ injection by ChIP assay $(n=3-5)$. Rhythmic pattern of VDR recruitment in Bmal1 ${ }_{\mathrm{lnt}}^{\mathrm{fl} / \mathrm{fl}}$ mice was not detected in Bmal1${ }_{\mathrm{lnt}}{ }^{-1-}$ mice. ${ }^{*} P<0.001,{ }^{* *} P<0.01,{ }^{* * *} P<0.05$ by 1 -way ANOVA.

analysis revealed the presence of E-boxes in the first intron, luciferase assays failed to reveal functional E-boxes in this region (Supplemental Figure 3D). Because the VDRE resides in the Vdr gene (23), we speculated that the rhythmic recruitment of VDR to the VDRE of the $V d r$ gene created the rhythmic expression of $V d r$. In support of this, VDR was recruited to the VDRE in the $V d r$ gene in a time-dependent manner, with greater recruitment at ZT8 (Figure 2C).

Clock physically interacts with VDR and facilitates VDR transcriptional activity. We investigated the molecular mechanisms by which the lack of Bmall disrupted the rhythmic recruitment of VDR to the VDRE. 


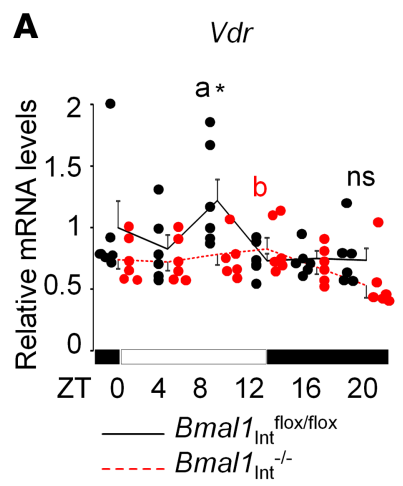

C

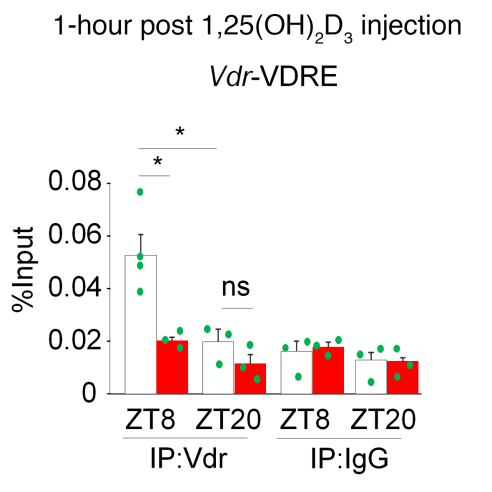

$B m a / 1_{\text {Int }}^{\text {flox/flox }}$
B $\quad \mathrm{HC}$ for Vdr

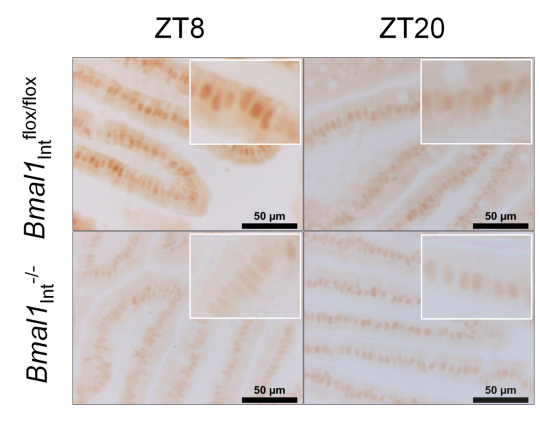

4-hour post $1,25(\mathrm{OH})_{2} \mathrm{D}_{3}$ injection Vdr-VDRE

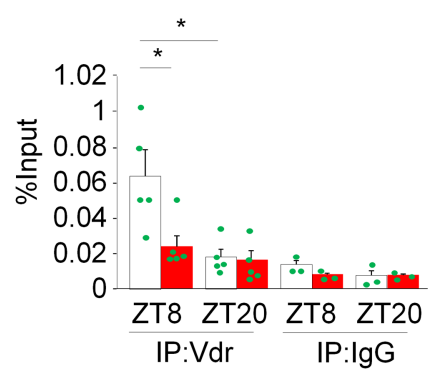

$B m a l 1_{\operatorname{lnt}}^{-1-}$

Figure 2. VDR is rhythmically recruited at the VDRE of the Vdr gene. (A) Villi were collected from the duodenum at 8 weeks of age every 4 hours, and expression of Vdr was determined by real-time RT-PCR $(n=6)$. (a) $P<0.05$, ZT8 vs. ZT4; $P<0.01$, ZT8 vs. ZT12, ZT16, and ZT20, in Bmal1 ${ }_{\text {Int }}^{\text {flfl }}$ mice, by 1-way ANOVA. (b) $P<0.01, \mathrm{ZT12}$ vs. ZT20; in Bmal1 $_{\text {Int }}{ }^{-1-}$ mice, by 1-way ANOVA. ${ }^{*} P<0.01$, Bmal1 $_{\text {Int }}{ }^{f / f l}$ vs. Bmal1 ${ }_{\text {Int }}{ }^{-1-}$ mice at ZT8 by Student's $t$ test. (B) VDR protein expression in the duodenum of 8-week-old mice was determined by immunohistochemistry. A representative of 3 independent experiments is shown. (C) Recruitment of VDR at the VDRE of the Vdr gene was analyzed 1 and 4 hours after $1,25-(\mathrm{OH})_{2} \mathrm{D}_{3}$ injection by ChIP assay $(n=3-5) .{ }^{*} P<0.05$ by 1 -way ANOVA.

A coimmunoprecipitation analysis revealed that VDR physically interacted with Clock, but not with Bmall (Figure 3A). When exogenous Bmall was coexpressed, the interaction between VDR and Clock was decreased, suggesting that the Clock interaction with VDR or Bmall is mutually exclusive (Figure 3A). A ChIP analysis performed 4 hours after 1,25-(OH) ${ }_{2} \mathrm{D}_{3}$ injection confirmed that Clock was recruited to the VDRE in the Vdr, Trpv6, and Cyp24a1 genes in a time-dependent manner, with greater recruitment at ZT8 in control mice, and this was not noted in Bmall $_{\mathrm{Int}}^{-1-}$ mice (Figure 3B). A luciferase assay revealed that Clock enhanced VDRE-mediated VDR transcriptional activity in the presence of Bmall (Figure 4A), whereas Clock alone failed to do so (Figure 4B), suggesting that Bmal1 is required for Clock to activate VDRE-mediated VDR transcriptional activity. In support of this notion, Clock was localized in the cytosol when Bmal1 was not coexpressed, whereas Clock was localized in the nucleus in the presence of Bmal1 (Figure 4C). Consistent with this, nuclear expression of Clock was decreased in Bmall-deficient mouse embryonic fibroblasts (Supplemental Figure 4A). In addition, Clock expression in the nucleus was stronger in control mice than Bmall $_{\text {Int }^{-/-}}$mice at ZT8 (Supplemental Figure 4B), suggesting that the lack of Bmal1 disrupts Clock-dependent VDR transcriptional activation during the light phase by impairing the nuclear transport of the Clock protein. Taken together, these findings indicate that after translocating into the nucleus with the assistance of Bmal1, Clock dissociates from the Bmal1/Clock complex and activates VDR transcriptional activity by forming a protein complex with VDR (Figure 4D).

Clock accelerates the acetylation of lysines in histone H4 proteins at the VDRE. In order to elucidate the molecular mechanisms by which Clock accelerates the binding of VDR to the VDRE, we tested the hypothesis that Clock allows chromatin to be transcriptionally permissive at VDR target genes. We analyzed the acetylation of lysines 5, 8, 12, and 16 in histone H4 at VDR target genes using a ChIP assay and found that 
A

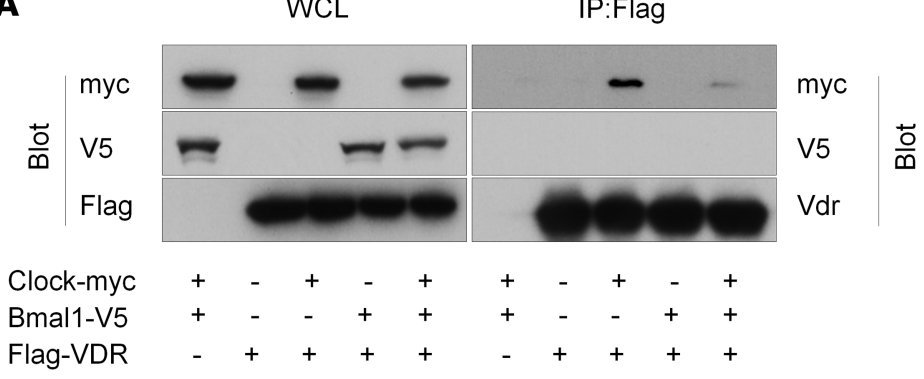

B

Dbp-Ebox
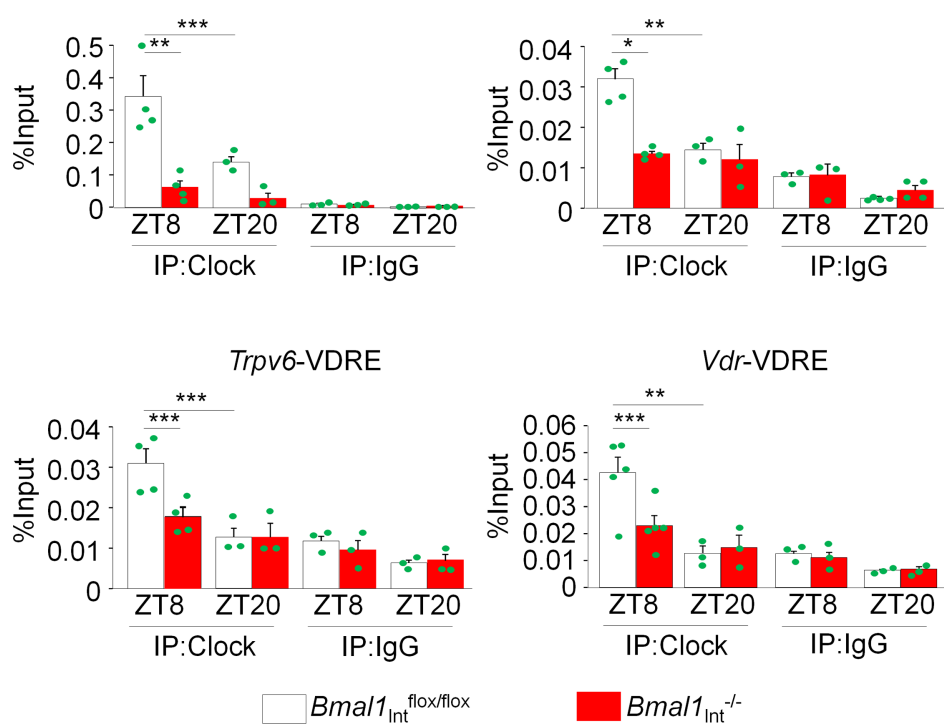

Figure 3. Clock physically interacts with VDR. (A) Physical interaction of Clock with VDR was evaluated using coimmunoprecipitation. Clock physically forms a protein complex with VDR. A representative of 3 independent experiments is shown. (B) Recruitment of Clock at the VDRE of the Cyp24a1, Trpv6, and Vdr genes was analyzed by ChIP assay ( $n=3-5)$. E-box of the $D b p$ gene was used as a positive control for Clock recruitment. ${ }^{*} P<0.001,{ }^{* *} P<0.01$, ${ }^{* * *} P<0.05$ by 1-way ANOVA.

acetylated H4 showed a rhythmic pattern, with ZT8 being greater than ZT20 in control mice, whereas this was absent in Bmall ${ }_{\text {Int }}{ }^{-1-}$ mice (Figure 5A). The Per1 E-box and Dbp E-box were used as positive controls for experiments. In order to clarify the underlying mechanisms, we examined whether the intrinsic histone acetyltransferase (HAT) activity of Clock was involved in this regulation (24). We forced-expressed wildtype Clock (WT-Clock) or the HAT activity-dead Clock mutant (MT-Clock) in HEK293T cells together with VDR and Bmall expression vectors and analyzed acetylated H4 levels at VDR target genes, such as $V D R$ and CYP24A1. As shown in Figure 5B, acetylated $\mathrm{H} 4$ levels were increased by the forced expression of WT-Clock, whereas that of MT-Clock did not affect its levels. The PER1 E-box was used as a positive control for experiments. We also investigated the involvement of other HATs, such as CREB-binding protein (CBP), because Clock has been shown to form a protein complex with CBP; however, we failed to detect the recruitment of CBP to the VDR/Clock protein complex (Supplemental Figure 5).

Transcellular calcium absorption in the duodenum is impaired in Bmall ${ }_{\text {Int }}{ }^{-1-}$ mice. Consistent with the expression profiles of VDR target genes, transcellular $\mathrm{Ca}$ absorption in control mice displayed a rhythmic pattern, with peaks at ZT8, and Bmall $_{\text {Int }}{ }^{-1-}$ mice showed reductions in transcellular Ca absorption, particularly at ZT8 and ZT14 (Figure 6A). We then analyzed the systemic consequences of impaired transcellular $\mathrm{Ca}$ absorption in Bmall $_{\text {Int }}{ }^{-1-}$ mice. Serum Ca concentrations exhibited a circadian profile with significant decreases during the dark phase in control mice, whereas rhythmic profiles were not observed in Bma$l 1_{\mathrm{Int}}{ }^{-1-}$ mice (Figure 6B). The serum concentration of Ca was not decreased in $B$ mall $_{\mathrm{Int}}^{-1-}$ mice, indicating that compensatory mechanisms to maintain circulating $\mathrm{Ca}$ levels were operative (Figure 6B). In accordance with this, the urinary excretion of Ca was decreased and serum parathyroid hormone (PTH) levels 
A

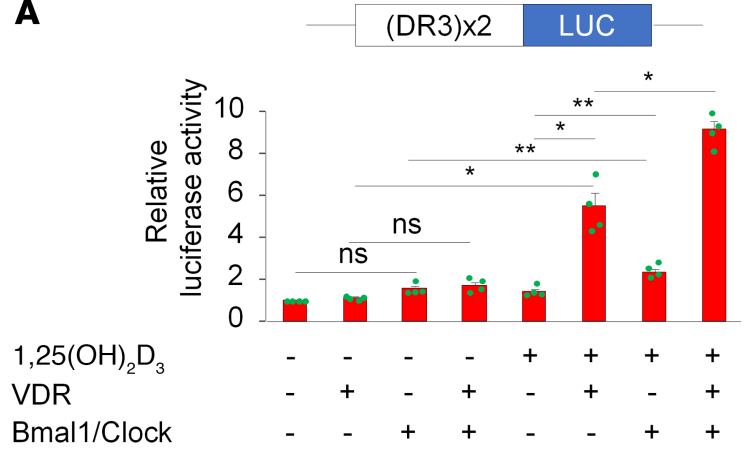

B

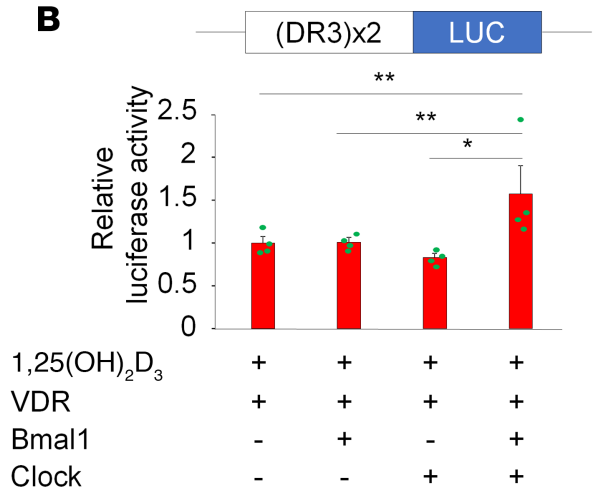

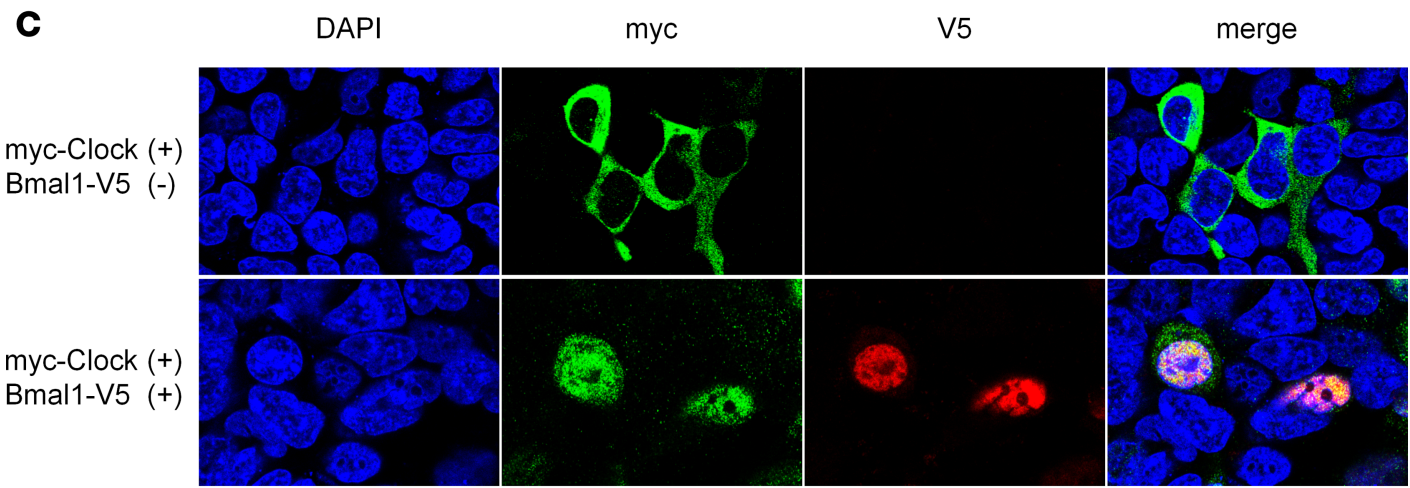

D

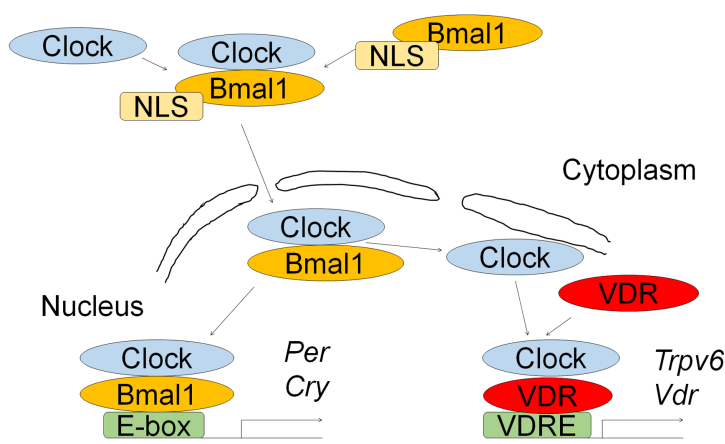

Figure 4. Bmal1 is required for Clock to activate VDR transcriptional activity. (A and B) Functional interaction of Clock with VDRE-mediated VDR transcriptional activity was determined by luciferase assay $(n=4) .{ }^{*} P<0.001,{ }^{* *} P<0.05$ by 1-way ANOVA. NS, not significant. (C) Myc-Clock and/or Bmal1-V5 was overexpressed in HEK293 cells and expression of Clock and Bmal1 was visualized using myc and V5 antibodies, respectively. DAPI was used for nuclear staining. A representative of 3 independent experiments is shown. (D) Schematic model of Clock-mediated VDR transcriptional activation. After translocating into the nucleus in the presence of Bmal1, Clock dissociates from the Bmal1/Clock complex. The binding of Clock to VDR enhances the transcriptional activity of VDR through the VDRE. NLS, nuclear localization signal.

were increased in Bmall ${ }_{\text {Int }}{ }^{-1-}$ mice (Figure 6, C and D). The renal expression of Cyp27b1 and Cyp24a1 was increased and decreased during the dark phase, respectively, in a time-dependent manner in Bmall $_{\mathrm{Int}^{-1}}$ mice $^{-}$ (Supplemental Figure 6), and, consistent with this result, circulating $1,25-(\mathrm{OH})_{2} \mathrm{D}_{3}$ levels were elevated in Bmall $_{\text {Int }}^{-1-}$ mice (Figure 6E). Furthermore, serum C-telopeptide (CTX) levels, a marker for bone resorption, were increased in Bmall $_{\text {Int }}^{-/-}$mice (Figure $6 \mathrm{~F}$ ), suggesting that bone resorption is activated as a compensatory mechanism for decreases in $\mathrm{Ca}$ absorption.

Bone mass is decreased in Bmal1 ${ }_{\text {Int }}^{-1-}$ mice. The above results prompted us to speculate that bone mass is decreased in $B_{m a l l}{ }_{\mathrm{Int}}{ }^{-1}$ mice. In order to test this, we performed a histomorphometric analysis of the femur and found that the trabecular ratio of bone volume to total volume (BV/TV) was reduced in Bma$l 1_{\text {Int }}{ }^{-1-}$ mice, and this was associated with increases in bone resorption parameters (Figure 7, A and B). $\mu \mathrm{CT}$ analysis also revealed the decreased trabecular bone mass of the femur in Bmall $_{\mathrm{Int}^{-1}}{ }^{-1}$ mice (Figure 7C). 

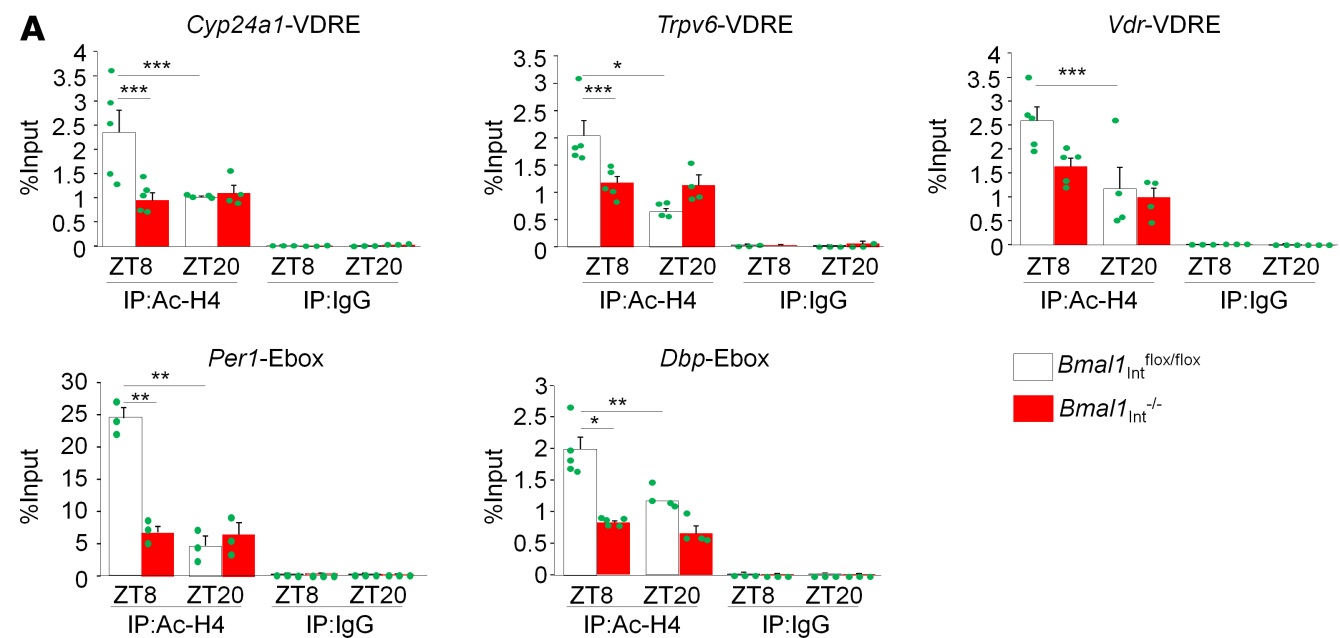

B

VDR-VDRE
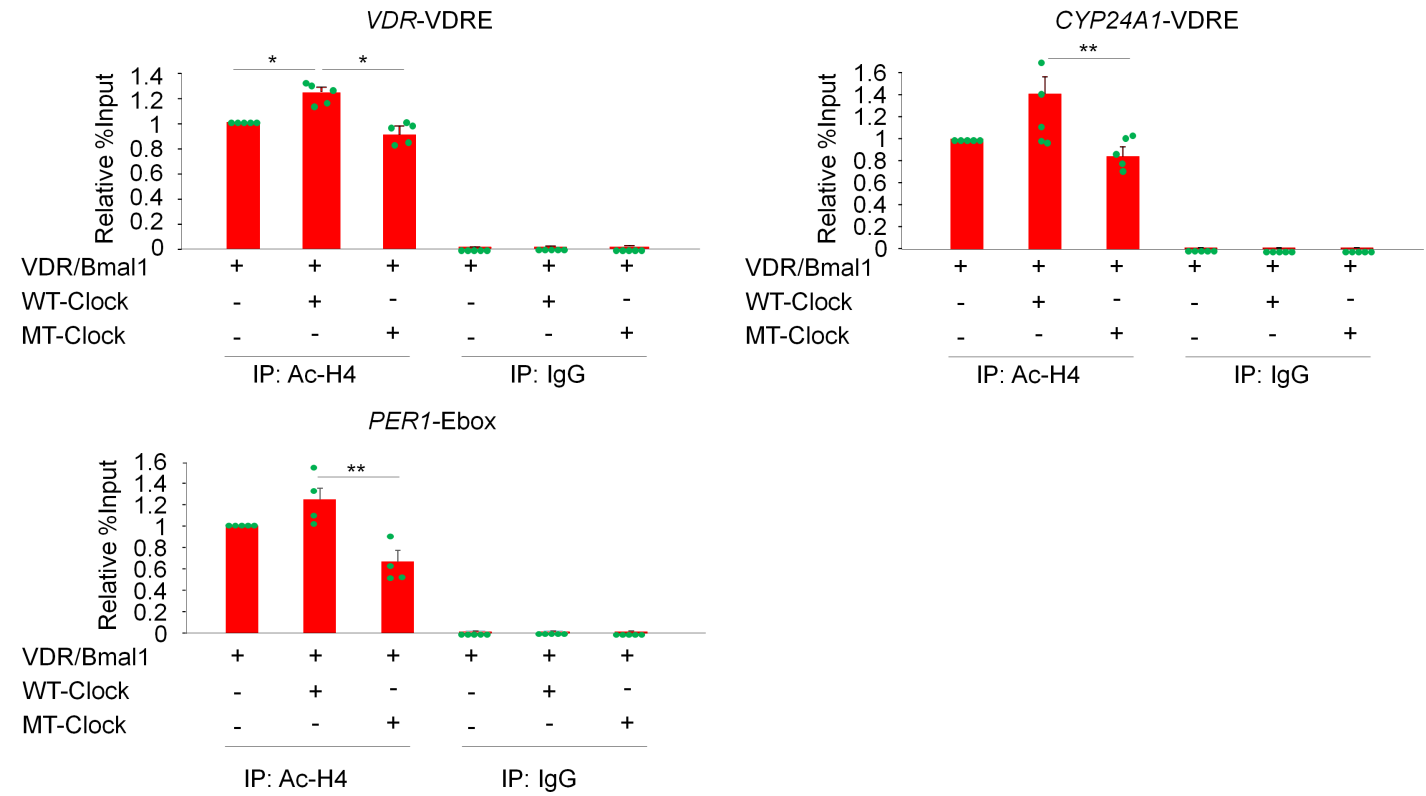

Figure 5. Clock enhances acetylated histone H4 levels at VDR target genes. (A) Acetylation of histone H4 (Ac-H4) at the VDRE of Cyp24a1, Trpv6, and Vdr genes was analyzed by ChIP assay $(n=3-5)$. E-boxes of the $D b p$ and $P e r 1$ genes were used as positive controls for experiments. ${ }^{*} P<0.001,{ }^{* *} P<0.01,{ }^{* * *} P$ $<0.05$ by 1 -way ANOVA. (B) The acetylation of histone $\mathrm{H} 4$ at the VDRE of VDR and CYP24A1 was analyzed by ChIP assay in HEK293T cells $(n=3-5)$. E-box of the PER1 gene was used as a positive control for experiments. ${ }^{*} P<0.01,{ }^{* *} P<0.05$ by 1 -way ANOVA.

Cortical thickness of the midshaft of the femur and trabecular bone mass of the lumbar vertebrae were not significantly affected (Supplemental Figure 7, A and B). The Rankl/Opg ratio was increased in Bmall Int $^{-1-}$ mice due to decreases in $O p g$ expression (Figure 7D). Ctpk expression also increased in Bmall ${ }_{\text {Int }}{ }^{-1}$ mice in a time-dependent manner (Figure 7D). Unexpectedly, bone formation markers, such as Ocn expression, the osteoblast number, and bone formation rate, were decreased in Bmall $_{\mathrm{Int}^{-1-}}$ mice, suggesting that the lack of Bmall in the intestines caused the uncoupling of bone formation and resorption (Figure 7, B and D). Since osteoblastogenesis of primary calvarial osteoblasts and osteoclastogenesis of spleen mononuclear cells from Bmall $_{\text {Int }}{ }^{-1-}$ mice did not show any significant differences from control cells, any excision of the Bmall gene in skeletal cells played a negligible role in the development of the osteopenic phenotype in Bma$11_{\text {Int }}{ }^{-1-}$ mice (Supplemental Figure 8, A-E). To test whether the low-bone-mass phenotype in $B$ mall $1_{\text {Int }}^{-1-}$ mice was caused by decreases in transcellular $\mathrm{Ca}$ absorption, mice were fed high-Ca diet $(\mathrm{HCaD})$ to minimize the effect of transcellular $\mathrm{Ca}$ absorption by enhancing paracellular $\mathrm{Ca}$ absorption. As shown in Figure 7C, $\mathrm{HCaD}$ caused increases in bone mass both in control and Bmall $_{\text {Int }}{ }^{-1-}$ mice, and a low-bone-mass phenotype in Bmall $_{\text {Int }}{ }^{-1-}$ mice was not noted under $\mathrm{HCaD}$. These findings may at least partially support the notion that decreases in transcellular Ca absorption contribute to reduced bone mass in $\mathrm{Bmall}_{\mathrm{Int}}{ }^{-1-}$ mice. 

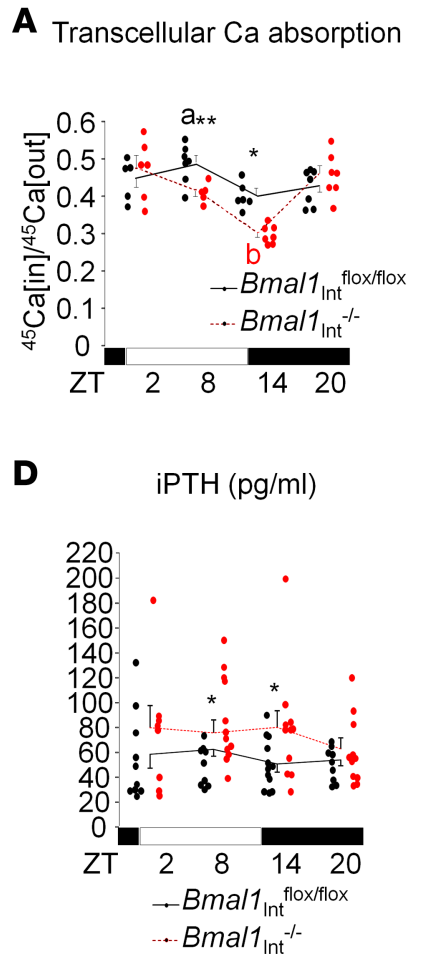
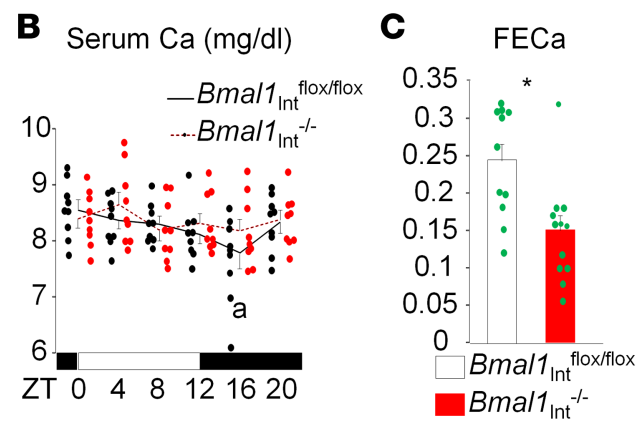

\section{E}

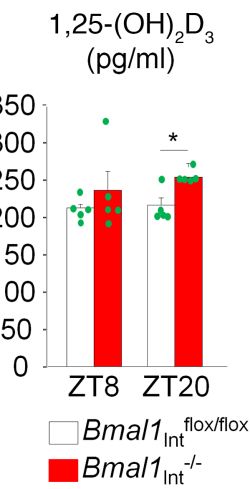

F $\quad \operatorname{CTX}(\mathrm{ng} / \mathrm{ml})$

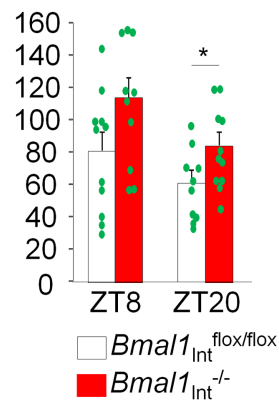

Figure 6. Transcellular Ca absorption is decreased in Bmal1 ${ }_{1_{\mathrm{Int}}}{ }^{-1}$-mice. (A) Transcellular Ca absorption was evaluated by everted gut sac assay at 8 weeks of age $(n=5-8)$. (a) $P<0.05$, ZT8 vs. ZT20; $P<0.01$, ZT8 vs. ZT14; in Bmal1 fl/fl mice, by 1-way ANOVA. (b) $P<0.01$, ZT14 vs. ZT8; $P<0.001$, ZT14 vs. ZT2 and ZT20; in Bmal1 ${ }^{-1-}$ mice, by 1-way ANOVA. ${ }^{*} P<$ $0.001,{ }^{* *} P<0.05 ; B$ mal1 ${ }_{\text {Int }}^{\text {flf }}$ vs. Bmal1 ${ }_{\text {Int }}{ }^{-1-}$ at indicated time points by Student's $t$ test. (B) Serum Ca levels were determined every 4 hours at 8 weeks of age $(n=9)$. (a) $P<0.05$, ZT16 vs. ZT4, ZT8, and ZT20; $P<0.01$, ZT16 vs. ZT0; in $B m a-$ $11^{\mathrm{fl} / \mathrm{fl}}$ mice, by 1-way ANOVA. (C) Urine volume and Ca excretion in the urine was measured at 8 weeks of age ( $n=11$ or 12). ${ }^{*} P<0.01$ by Student's $t$ test. FECa, fractional excretion of Ca. (D) Serum intact PTH $(n=8-12)$ levels were determined at 8 weeks of age. ${ }^{*} P<0.05 ; B$ mal1 ${ }_{\operatorname{lnt}}^{f l / f l}$ vs. Bmal1 ${ }_{\mathrm{lnt}}{ }^{-1-}$ mice at indicated time points by Student's $t$ test. (E and $\mathbf{F}$ ) Serum $1,25-(\mathrm{OH})_{2} \mathrm{D}_{3}(n=5)(\mathbf{E})$, and CTX $(n=10)(\mathbf{F})$ levels were determined at 8 weeks of age. ${ }^{*} P<0.05$ by Student's $t$ test.

Blockade of $\beta$-adrenergic signaling reversed impaired bone formation and partially rescued the osteopenic phenotype of Bmal1 ${ }_{\text {Int }}^{-1-}$ mice. These results demonstrated that the lack of Bmall in the intestines impaired transcellular $\mathrm{Ca}$ absorption and reduced bone mass by driving bone resorption; however, it currently remains unclear why bone formation is decreased in $B m a l 1_{\text {Int }}{ }^{-1-}$ mice. Therefore, we tested the hypothesis that the lack of Bmal1 in the intestines regulated bone metabolism in a manner involving neuronal circuits. In support of this, sympathetic activity, the activation of which is known to decrease bone mass by activating bone resorption and suppressing bone formation, was elevated in $\mathrm{Bmall}_{\text {Int }}{ }^{-1-}$ mice during the dark phase (Figure 8A). Since sympathetic activation disappeared in vagotomized $\mathrm{Bma}$ $11_{\mathrm{Int}}{ }^{-/-}$mice, the afferent vagal nerve is responsible for sympathetic activation in Bmall $_{\text {Int }}{ }^{-/-}$mice (Figure $8 \mathrm{~B})$. The efficacy of vagotomy was assessed by an evaluation of cholecystokinin octapeptide-induced (CCK-8-induced) satiety and alterations in body weight (Supplemental Figure 9, A and B). The activation of sympathetic activity was not likely due to increased synthesis of serotonin, which is known to mediate its signal from the duodenum to sensory neurons, since a rate-limiting enzyme for serotonin synthesis, tryptophan hydroxylase (Tph1), was not affected by the lack of Bmall in the duodenum (Figure 8C). Additionally, the gut innervation in the duodenum was not affected either (Figure 8D). We finally tested the idea that the lack of Bmall increased sympathetic tone through the modulation of microbiota. For this purpose, mice were treated with antibiotics and sympathetic tone was determined. As shown in Figure 8E, sympathetic tone was elevated in Bmall $_{\mathrm{Int}}{ }^{-1-}$ mice even in the presence of antibiotics compared with antibiotic-treated control mice, suggesting that the modulation of microbiota is unlikely the cause of enhanced sympathetic tone in $B m a l 1_{\mathrm{Int}}^{-1-}$ mice. These findings suggest that increases in sympathetic tone in Bmall $_{\text {Int }}{ }^{-/}$mice are independent of alterations in serotonin synthesis, gut innervation, or gut microbiota. 
A
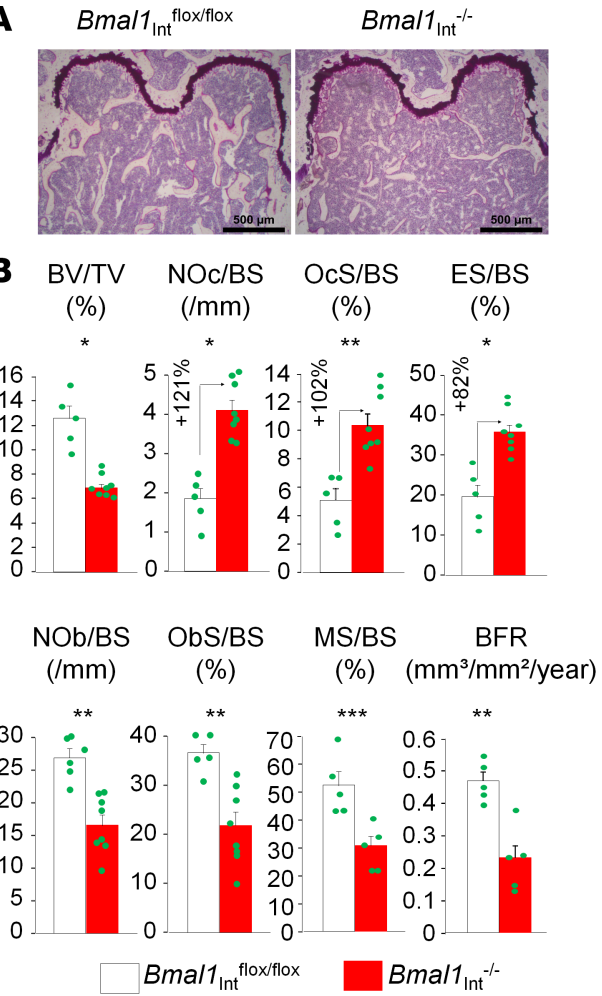

C

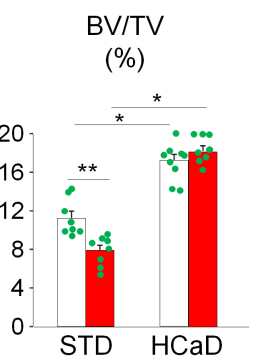

D
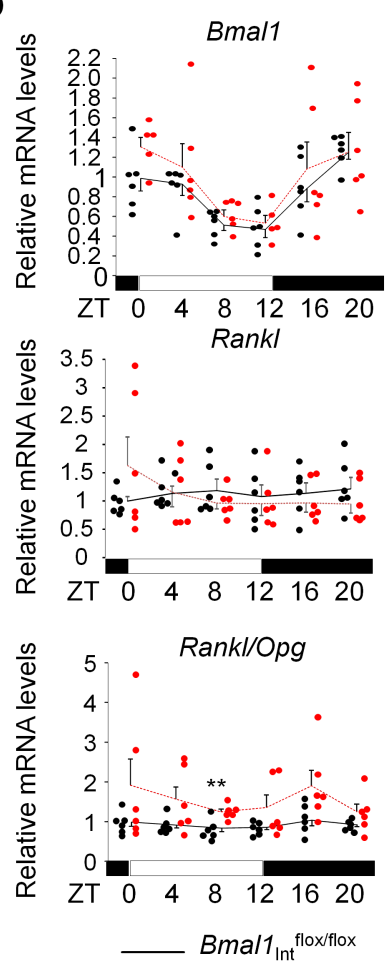

Ocn
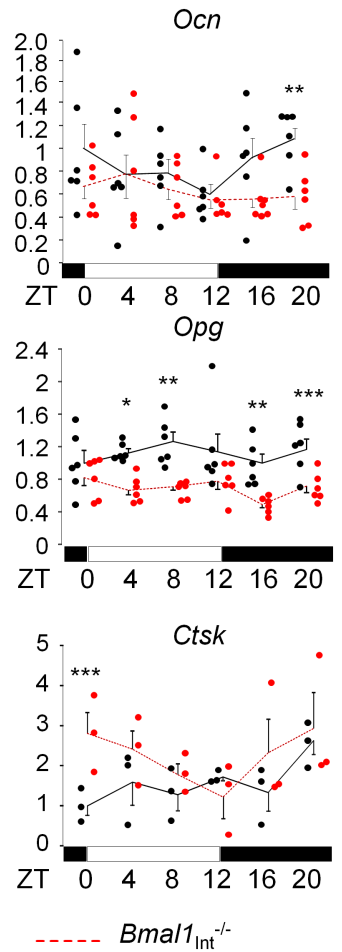

Figure 7. Bone mass is reduced in Bmal1 ${ }_{\text {Int }}{ }^{-1-}$ mice and associated with increases in bone resorption and decreases in bone formation. (A) Villanueva bone staining of the distal femur of 16-week-old mice. A representative of at least 3 independent experiments is shown. NOc, number of osteoclasts; BS, bone surface; ES, eroded surface; Nob, number of osteoblasts; ObS, osteoblast surface; MS, mineralizing surface; BFR, bone formation rate. (B) Dynamic histomorphometry of the femur was performed at 16 weeks of age. ${ }^{*} P<0.001,{ }^{* *} P<0.01,{ }^{* *} P<0.05$ by Student's $t$ test. The difference in the values of bone resorption markers between control and Bmal1 ${ }_{\mathrm{lnt}}{ }^{-1-}$ mice is shown. (C) Mice were fed standard chow (STD) or high-Ca diet (HCaD) starting at 4 weeks of age for 12 weeks. Trabecular bone microarchitecture of the distal femur was evaluated by $\mu \mathrm{CT}$ analysis $(n=8-10)$. ${ }^{*} P<0.001,{ }^{* *} P<0.01$, , ${ }^{* *} P<0.05$ by 1 -way ANOVA. (D) Femurs were collected from 8-week-old mice every 4 hours, and the expression of the genes of interest was examined by real-time RT-PCR ( $n$ = 6). ${ }^{*} P<0.001,{ }^{* *} P<0.01,{ }^{* * *} P<0.05 ; B m a / 1_{\text {Int }}{ }^{f / f l}$ vs. Bmal1 ${ }_{\mathrm{Int}}{ }^{-1-}$ at indicated time points by Student's $t$ test.

In order to clarify whether the activation of sympathetic tone is responsible for bone loss in Bma$11_{\text {Int }}{ }^{-/}$mice, mice were treated with propranolol (PRO), a $\beta$-blocker. As shown in Figure 9, A and B, bone loss in Bmal1 $_{\text {Int }}^{-/-}$mice was partially rescued by the treatment with PRO, with bone formation markers being similar between controls and Bmal1 ${ }_{\mathrm{Int}}^{-/-}$mice in the presence of PRO (Figure 9C). Consistent with this, serum procollagen type I N propeptide (P1NP) levels, a bone formation marker, were decreased in Bmall $_{\text {Int }}{ }^{-1-}$ mice compared with controls, whereas this difference disappeared in the presence of PRO (Figure 9D). Although bone resorption markers were still higher in $\mathrm{Bmall}_{\mathrm{Int}}{ }^{-1-}$ mice than in the controls under PRO treatment, the extent of increases in bone resorption markers in the presence of PRO was smaller than those in the absence of PRO (compare Figure 9C with Figure 7B), suggesting that blockade of sympathetic tone partially suppressed bone resorption in $\mathrm{Bmall}_{\mathrm{Int}}{ }^{-1-}$ mice. These results indicate that the lack of Bmal1 in the intestines suppresses bone formation and activates bone resorption, in part by driving sympathetic tone, and decreases bone mass (Figure 9E). 
A

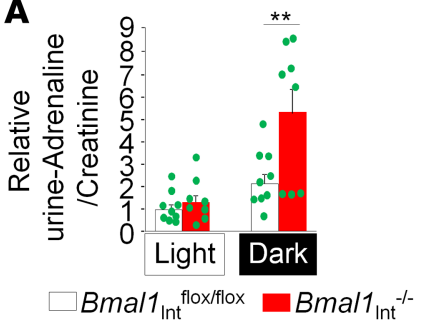

B

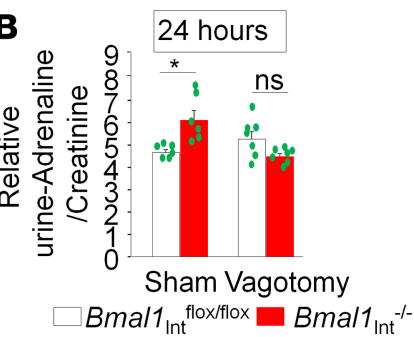

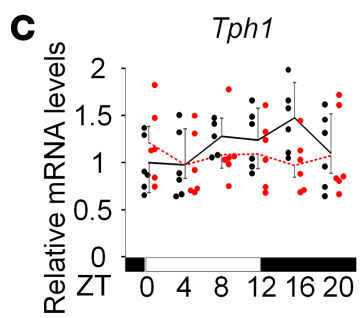

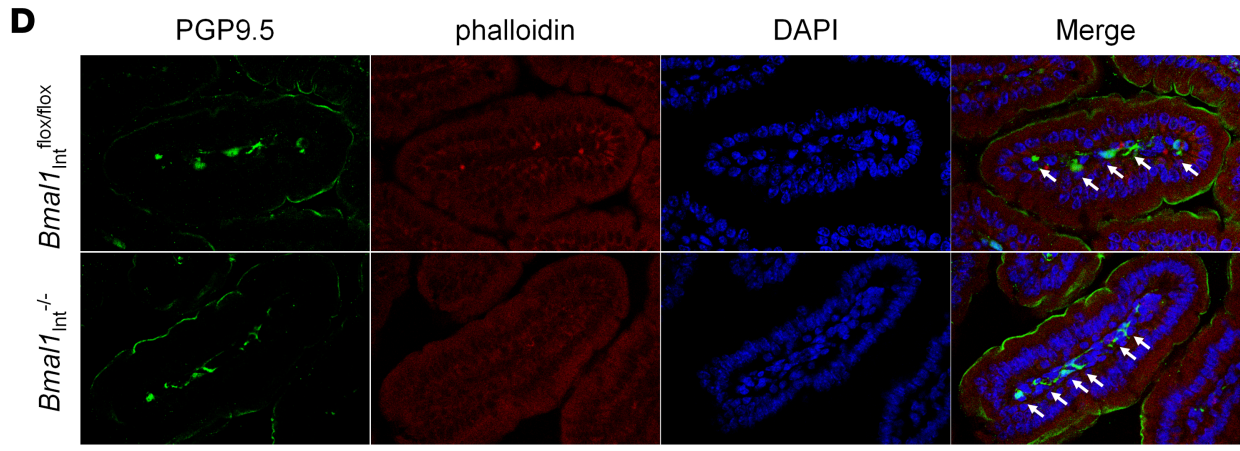

$\mathbf{E}$

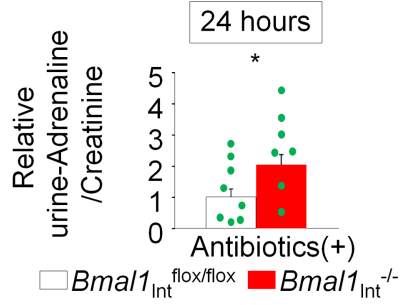

Figure 8. Sympathetic tone is elevated in Bmal1 ${ }_{\text {Int }}^{-/-}$mice. (A and B) Sympathetic activity was evaluated by measuring urine adrenaline levels. (A) Sympathetic activity was elevated in $B m a / 1_{\mathrm{Int}}{ }^{-1-}$ mice during the dark phase $(n=9)$. (B) Increases in sympathetic activity in sham-operated $B$ mal1 ${ }_{\mathrm{Int}}^{-1-}$ mice were not noted in vagotomized $B$ mal1 ${ }_{\mathrm{Int}}{ }^{-1-}$ mice $(n=6$ or 7$){ }^{*} P<0.01$, ${ }^{*} P<0.05$ by 1 -way ANOVA. (C) Villi were collected from the duodenum at 8 weeks of age every 4 hours, and expression of Tph1 was determined by real-time RT-PCR $(n=6)$. (D) Expression of PGP9.5 in the duodenum was visualized by immunofluorescence. A representative of 3 experiments is shown. (E) Mice were treated with antibiotics for 4 weeks starting at 10 weeks of age, and 24-hour urine samples were collected. Urine levels of adrenaline were evaluated $(n=7$ or 8$)$. ${ }^{*} P<0.05$.

\section{Discussion}

Accumulating evidence has demonstrated that skeletal metabolism is regulated in a manner involving the circadian clock network, as partly demonstrated by clinical findings showing that circulating bone formation and resorption markers exhibit circadian profiles (25). Mice in which the circadian clock network is disrupted show a skeletal phenotype, supporting the link between the circadian clock system and skeletal metabolism; however, the exact role of the peripheral circadian clock network on skeletal metabolism is context specific and has yet to be elucidated in detail (14-16). In an attempt to clarify the underlying mechanisms, we investigated the circadian regulation of $\mathrm{Ca}$ homeostasis because $\mathrm{Ca}$ is a fundamental nutritional factor regulating skeletal metabolism. Because there is accumulating evidence showing that the circadian network in the intestines plays important roles in the regulation of metabolic homeostasis, including nutrient absorption, we examined intestinal tissue as a nodal organ integrating the circadian network and $\mathrm{Ca}$ homeostasis. In line with this, the expression of genes involved in transcellular $\mathrm{Ca}$ absorption exhibited circadian profiles in the duodenum and this was associated with the rhythmic profile of transcellular $\mathrm{Ca}$ absorption. Serum $\mathrm{Ca}$ levels showed a rhythmic pattern in control mice, with the lowest levels being observed during the dark phase when mice behave and eat actively, and this result is consistent with decreases in Ca levels during the daytime in humans (13). In addition, serum Ca concentration showed circadian profiles in control mice and this disappeared in Bmal1 $_{\text {Int }}^{-/-}$mice. These results suggest that the intestinal clock is a primary system creating rhythmicity in circulating Ca levels. 
A

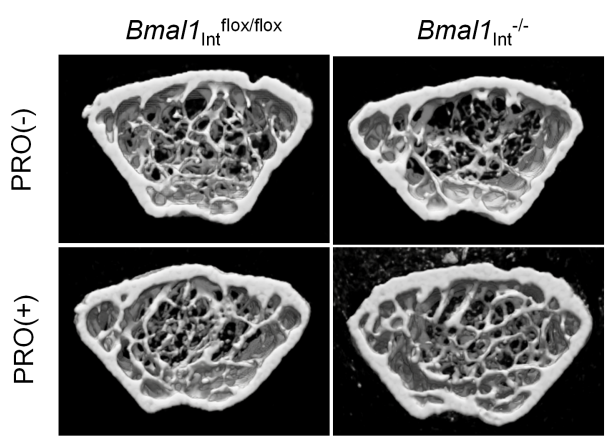

B

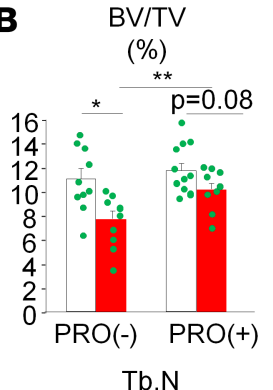

$(/ \mathrm{mm})$

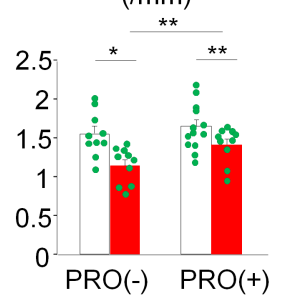

Tb.Th

$(\mu \mathrm{m})$
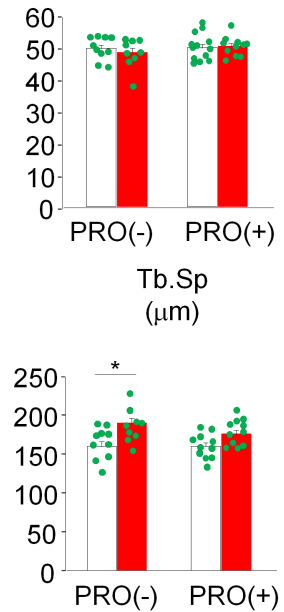

$\square B m a / 1_{\text {Int }}^{\text {flox fllox }} \quad B m a / 1_{\text {Int }^{-1-}}$

D Serum PINP levels (ng/ml)

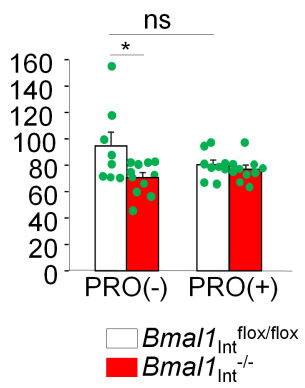

$\mathbf{E}$

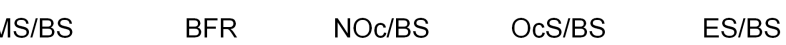

(\%) $\quad\left(\mathrm{mm}^{3} / \mathrm{mm}^{2} /\right.$ year $) \quad(/ \mathrm{mm})$

\section{(\%)}

$\mathrm{P}=0.16$

(\%)

$\mathrm{P}=0.07$

$+52 \%+43 \%$

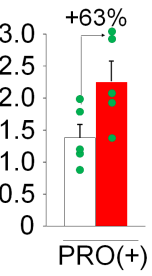

$\because$

40 : 0.25

0

$\mathrm{PRO}(+)$

Bmal1

$\mathrm{PRO}(+)$

30 -

20

10

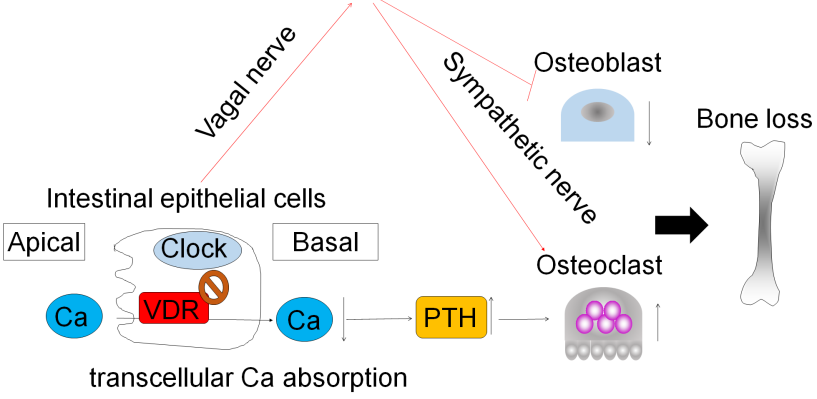

Figure 9. Sympathetic activation is partially responsible for bone loss in Bmal1 ${ }_{1 \mathrm{nt}}{ }^{-1-}$ mice. (A and B) $\mu \mathrm{CT}$ analysis of the femur was performed to analyze the trabecular bone microarchitecture of 16-week-old mice treated with or without propranolol (PRO). A representative $\mu \mathrm{CT}$ image (A) and the parameters of the $\mu \mathrm{CT}$ analysis are shown (B) $(n=10-13)$. ${ }^{*} P<0.01,{ }^{* *} P<0.05$ by 1 -way ANOVA. (C) Dynamic histomorphometric data of 16 -week-old mice treated with PRO $(n=5) .{ }^{*} P<0.05$ by Student's $t$ test. The difference in the values of bone resorption markers between control and Bmal1 $_{\text {Int }}{ }^{-1-}$ mice in the presence of PRO is shown, and this is smaller than those in the absence of PRO (see Figure 7B). (D) Serum levels of P1NP were determined at ZT2 $(n=8-11)$. (E) Schematic model of the effects of the clock network in the intestines on skeletal metabolism. The lack of Bmal1 in the intestines blunts the Clock-mediated activation of VDR transcriptional activity, which results in decreases in transcellular Ca absorption. This leads to osteoclastic activation and bone loss. Additionally, sympathetic tone is activated through afferent vagal nerves when Bmal1 is deleted in the intestines, which leads to bone loss through the suppression of bone formation and stimulation of bone resorption.

We next investigated the mechanisms by which the circadian profiles of genes involved in transcellular $\mathrm{Ca}$ absorption are regulated. Since transcellular $\mathrm{Ca}$ absorption is mainly regulated in a manner involving the VDR signaling pathway (26), we hypothesized that Bmall/Clock regulated rhythmicity in the expression of $V d r$, but failed to detect the presence of E-boxes in the $V d r$ gene locus. These results prompted us to speculate 
that the circadian network regulates transcellular $\mathrm{Ca}$ absorption through its modulation of the function of VDR. Indeed, Clock functionally and physically interacted with VDR and accelerated the binding of VDR to the VDRE in a circadian-dependent manner. Because the nuclear localization of Clock occurred in a circadian-dependent manner with stronger nuclear expression during the light phase $(27,28)$, rhythmicity in VDR target genes may be generated in a manner that is dependent on cyclic nuclear Clock localization. Interestingly, the binding of Clock to VDR was independent of Bmal1, as was described in the case of Bmall's interaction with NF- $\mathrm{KB}$ (29). In addition, because of the lack of a nuclear localization signal in the Clock protein (22), binding with Bmal1 is necessary for Clock to translocate into the nucleus. These findings suggest a unique role for Clock such that after translocating into the nucleus with the assistance of Bmal1, Clock dissociates from the Bmal1/Clock complex and functions as a coactivator (Figure 4D); however, further studies are required in order to confirm this speculation.

Since histone acetylation plays pivotal roles in transcriptional activation (30), we investigated whether the binding of Clock to VDR enhanced the acetylation of the lysine residues of histone proteins at VDR target genes. In line with previous findings showing that Clock exhibits intrinsic HAT activity $(24,31,32)$, our results also suggested the involvement of the intrinsic HAT activity of Clock in histone acetylation at the VDRE. Because Clock has been shown to physically interact with other HATs, such as CBP/p300, PCAF, and TIP60 (24), we also examined the hypothesis that Clock recruited CBP and accelerated histone acetylation at the VDRE, but failed to detect the formation of the VDR/Clock/CBP protein complex. Numerous HATs have been reported to form a protein complex with VDR, such as SRC1; therefore, we cannot exclude the possibility of the recruitment of HATs other than CBP. Nevertheless, the present results provide evidence to show that Clock accelerates histone acetylation and stimulates the transcriptional activity of its binding partner.

Surprisingly (because bone formation is generally coupled to bone resorption), the observation that bone formation parameters were not elevated in $\mathrm{Bmall}_{\mathrm{Int}}{ }^{-/-}$mice despite enhanced bone resorption suggested that the lack of Bmall in the intestines impaired bone formation independently of its regulation in Ca homeostasis. Because accumulating evidence has clearly demonstrated a critical role for the neuronal network in skeletal metabolism, we investigated neuronal involvement in this regulation. Based on evidence showing that sympathetic activation reduces bone mass by suppressing bone formation and stimulating bone resorption (33), we assessed sympathetic activity and found that it was elevated in Bmall $_{\text {Int }}{ }^{-1-}$ mice and disappeared when vagal nerves were dissected. We investigated the molecular mechanisms by which the disruption in the intestinal circadian network activates sympathetic activity. First, we analyzed the expression of $T p h 1$, a rate-limiting enzyme for serotonin synthesis, because serotonin has been shown to mediate the signals from the duodenum to sensory neurons, but failed to detect any differences in its expression between control and Bmal1 $_{\text {Int }}^{-1-}$ mice (34). Second, we analyzed the gut innervation because the communication between gut and neuron has recently gained more attention among researchers (35). For this purpose, we performed PGP9.5 staining but the innervation between control and Bmall $_{\text {Int }}^{-1-}$ mice was not different. Third, we investigated the involvement of gut microbes in this regulation because accumulating evidence has demonstrated the critical roles of gut microbiota in the regulation of the afferent nervous system from the gut (36); however, experiments based on antibiotic treatment revealed that gut microbiota was unlikely involved in this regulation. In sum, these results suggest that there is a functional link between the intestinal circadian system and skeletal metabolism though the neuronal network; however, the molecular mechanisms by which the intestinal clock mediates its signals to the neuronal pathway have yet to be elucidated.

In summary, we herein provided evidence to show that the circadian clock network in the intestines regulates skeletal metabolism through the regulation of $\mathrm{Ca}$ homeostasis and sympathetic activity. A number of clinical studies have shown that the disruption of light/dark and/or sleep/wake cycles causes metabolic complications, including an increased incidence of obesity and cardiovascular complications (37); however, evidence for its influence on skeletal metabolism is limited. Therefore, these lines of evidence may add to our growing knowledge on the role of the circadian network in the regulation of $\mathrm{Ca}$ and skeletal metabolism and provide insights into the unrecognized function of the intestinal circadian system, which may be important for skeletal biology.

\section{Methods}

Animal studies. Bmal1 ${ }_{\text {Int }}^{\text {flft }}$ mice on a C57BL/6J background, which contain 2 loxP sites flanking exons 6 to 8 of the Bmal1 gene, were generated as previously reported (38). The excision of exons 6 to 8 results in the deletion of the bHLH domain and this is associated with the introduction of an early stop codon. Villin-Cre 
mice on a C57BL/6J background [B6.Cg-Tg(Vil1-cre)997Gum/J], which express Cre recombinase under the control of the Villin promoter, were purchased from Jackson Laboratories. Intestine-specific Bmal1-knockout

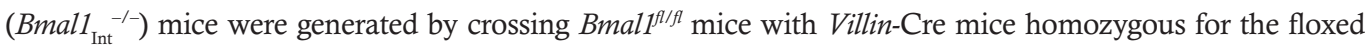
allele. Because the skeletal phenotype of Villin-Cre mice has been shown to be comparable to that of WT mice (39), Bmal1 ${ }^{f l f l}$ mice were used as controls for Bmall $_{\text {Int }}{ }^{-/-}$mice. The excision of the floxed allele was confirmed based on a PCR analysis using genomic DNA as described in Supplemental Methods. Mice were maintained on a 12-hour/12-hour LD cycle in a pathogen-free animal facility with free access to water and standard chow containing 1.0\% Ca and 1.0\% inorganic phosphate (Pi) (CE-2, CLEA Japan, Inc.). Where HCaD containing $2.4 \% \mathrm{Ca}$ and $1.0 \% \mathrm{Pi}$ (CLEA Japan, Inc.) was used, mice were switched to $\mathrm{HCaD}$ starting at 4 weeks of age. For antibiotic treatment, mice were treated with antibiotic-containing drinking water from 10 to 14 weeks old. Metronidazole (1 g/1) (Nacalai Tesque Inc.), ampicillin sodium (1 g/1) ( Nacalai Tesque Inc.), neomycin sulphate (1 g/1) ( Wako Pure Chemical Industries, Ltd.), and vancomycin hydrochloride (0.5 g/1) (Wako Pure Chemical Industries, Ltd.) were added to the drinking water.

In vivo ChIP assays. Control and Bmal1 $_{\text {Int }^{-1-}}$ mice at 8-9 weeks of age were intraperitoneally injected at ZT4 or ZT16 with either vehicle (propylene glycol; Wako Pure Chemical Industries, Ltd.) or 1,25-(OH) ${ }_{2} \mathrm{D}_{3}$ (EMD Millipore) at a dose of $10 \mathrm{ng} / \mathrm{g}$ of body weight, and 1 and 4 hours after the injection (ZT8 or ZT20) the intestinal villi from $7 \mathrm{~cm}$ of the proximal duodenum and jejunum were scraped by coverslips and ChIP was performed as described in Supplemental Methods.

In vitro ChIP assays. The expression vector containing the HAT activity-dead Clock mutant was generated based on a previous study (24) using QuikChange II XL (Agilent Technologies) according to the manufacturer's protocol. HEK293T cells were transfected with the pcDNA3.1-mBmal1-V5 vector, pSG5hVDR vector, and/or pFlag vectors containing WT-mClock (WT-Clock) or HAT activity-dead mClock (MT-Clock) using the PEI method. Six hours after treatment with $10^{-8} \mathrm{M} 1,25-(\mathrm{OH})_{2} \mathrm{D}_{3}$, cells were harvested and ChIP was performed as described in Supplemental Methods.

Luciferase assay. VDRE-mediated VDR transcriptional activity was analyzed using the luciferase vector [pGV-P2-(DR3) $)_{2}$ containing 2 tandem repeats of canonical DR3 (direct repeat spaced by 3 nucleotides). HEK293T cells were transfected with pGV-P2-(DR3) $2(0.2 \mu \mathrm{g})$ in combination with the pSG5-hVDR $(0.05 \mu \mathrm{g})$ expression vector, pcDNA3.1-mBmal1-V5 expression vector $(0.1 \mu \mathrm{g})$, and/or pSG5-mClock-myc expression vector $(0.1 \mu \mathrm{g})$, as indicated in the figures, and 24 hours after transfection, cells were treated with $10^{-8} \mathrm{M} 1,25-(\mathrm{OH})_{2} \mathrm{D}_{3}$ overnight, followed by the assessment of luciferase activity using specific substrates in a luminometer as described in Supplemental Methods.

Everted gut sac assay. Intestinal transcellular Ca transport was evaluated using the everted gut sac assay as reported by Benn et al., with modifications (40). Briefly, a 2-cm piece of the duodenum proximal to the pyloric junction was dissected from 8-week-old mice and an everted gut sac was prepared as previously reported (40). The intestinal sac was filled with $150 \mu \mathrm{l}$ of transport buffer ( $125 \mathrm{mM} \mathrm{NaCl}, 10 \mathrm{mM}$ fructose, $1.3 \mathrm{mM}$ HEPES, and $0.25 \mathrm{mM} \mathrm{CaCl}_{2}$ [pH 7.4]) and incubated in $10 \mathrm{ml}$ of transport buffer containing 45 $\mathrm{CaCl}_{2}(20,000 \mathrm{cpm} / \mathrm{ml})$ in a water bath at $37^{\circ} \mathrm{C}$ for 1 hour that was aerated continuously with $95 \% \mathrm{O}_{2} / 5 \%$ $\mathrm{CO}_{2}$. After this incubation, transport buffer collected from inside or outside the sacs was assayed in triplicate for ${ }^{45} \mathrm{Ca}$ using a scintillation counter.

Bilateral sub-diaphragmatic vagotomy and its assessment. Bilateral sub-diaphragmatic vagotomy was performed according to a previously reported method (41). Briefly, mice were deeply anesthetized, and a midline abdominal incision was made. The liver was pushed aside to allow for the visualization of the stomach and esophagus. Under a surgical microscope, the left and right vagal nerves running along the esophagus were excised. In sham-operated mice, the same surgical procedures, except for the excision of vagal nerves, were performed. In the assessment of vagotomy, the satiety effect of CCK-8 (Sigma-Aldrich) was investigated. Briefly, after 20 hours of fasting, sham-operated and vagotomized mice were intraperitoneally administered CCK- 8 at a dose of $8 \mu \mathrm{g} / \mathrm{kg} /$ body weight, and food intake was monitored for 1 hour. Theoretically, because CCK-8-induced satiety is mediated through the afferent vagal nerve (42), food intake after fasting is not observed in CCK-8-treated sham-operated mice, whereas vagotomized mice are resistant to the satiety effect of CCK-8.

$R N A$ isolation and quantitative real-time PCR. A $2-\mathrm{cm}$ piece of the duodenum proximal to the pyloric junction was dissected and villi were scraped by coverslips. Total RNA was isolated and real-time RT-PCR analysis was performed as described in Supplemental Methods. Gapdh was used as an internal standard control gene for all quantifications. 
Coimmunoprecipitation. The transfection of expression vectors in HEK293T cells was performed using the PEI method. Cells were solubilized in NP-40 buffer (5 mM EDTA, $150 \mathrm{mM} \mathrm{NaCl}, 0.5 \%$ Nonidet P-40, 10\% glycerol, and $10 \mathrm{mM}$ Tris-HCl, $\mathrm{pH}$ 8.0) containing a protease inhibitor cocktail (Complete TM, EDTA-free; Roche Diagnostics) and centrifuged. Supernatants were precleared with protein A/G-Sepharose (Santa Cruz Biotechnology) at $4^{\circ} \mathrm{C}$ for 2 hours, followed by immunoprecipitation with an anti-Flag antibody (Sigma-Aldrich, F3165) at $4^{\circ} \mathrm{C}$ overnight. Samples were washed 5 times with PBS containing a protease inhibitor cocktail and then analyzed by Western blot as described in Supplemental Methods.

Actogram. Eight-week-old mice were maintained individually in cages equipped with running wheels (RW-15; Melquest) with free access to water and food and housed for a week for adjustments. Wheel-running data were recorded using the cFDM-300 system (Melquest) and actograms were double-plotted using Feedam software (Melquest). Mice were kept under LD cycles for 10 days, switched to a DD cycle for an additional 10 days, and then returned to LD cycles.

$\mu C T$. The skeletal micro-architecture of the trabecular bones of the femur was analyzed by $\mu \mathrm{CT}$ (ScanXmate-RX; Comscantechno Co., Ltd.). Bones were scanned at an energy level of $70.5 \mathrm{kVp}$ and intensity of $115 \mu \mathrm{A}$. At the distal femur, trabecular bone was evaluated starting approximately $0.36 \mathrm{~mm}$ proximal to the femoral growth plate, and extending proximally $1.8 \mathrm{~mm}$. Measurements included BV/TV, the trabecular number (Tb.N), trabecular thickness (Tb.Th), and trabecular separation (Tb.Sp). All scans were analyzed using TRI/3D-BON software (RATOC System Engineering Co., Ltd.).

Histomorphometry. Histomorphometry was performed as previously described (43-45). In dynamic bone histomorphometry, 16-week-old male mice were labeled with subcutaneous injections of $16 \mathrm{mg} / \mathrm{kg}$ of calcein (Dojindo Co.) 96 and 24 hours before sacrifice. Femurs were collected from 16-week-old mice, and fixed in $70 \%$ ethyl alcohol. Undecalcified bone sections were prepared as follows: after staining with the Villanueva bone stain for 6 days, samples were dehydrated, defatted in an acetone/methyl-methacrylate monomer mixture (1:2), and embedded in methyl-methacrylate (Wako Pure Chemical Industries, Ltd.) without decalcification. Five-micrometer-thick longitudinal sections were prepared using a microtome (Leica), and bone histomorphometric measurements were obtained using a semiautomatic image analyzing system (System Supply) and fluorescence microscope (Olympus BX-53) set at a magnification of $\times 400$. Standard bone histomorphometrical nomenclatures, symbols, and units were used as described in the report of the ASBMR Histomorphometry Nomenclature Committee (46).

Statistics. All data are expressed as the mean \pm the standard error of the mean (SEM). Data were analyzed for significant differences by 2-tailed Student's $t$ test or 1-way ANOVA followed by Bonferroni's multiple comparison post hoc test. Significance was set at $P<0.05$.

Study approval. All animal studies were reviewed and approved by the Institutional Animal Care and Use Committee of Osaka Women's and Children's Hospital at Izumi, Osaka, Japan (board members: Isao Matsuo, Itaru Yanagihara, and Nobuhiko Okamoto).

\section{Author contributions}

MK conceived the project and designed the research. MK and SK performed most of the experiments. SS generated Bmalf $f^{f / f l}$ mice. All the authors analyzed the data. MK wrote the manuscript.

\section{Acknowledgments}

The authors thank K. Mochida and I. Matsuo (Osaka Women's and Children's Hospital) for generating specific pathogen-free mice. The authors are thankful to J. Kikuta (Osaka University Graduate School of Medicine), and M. Fujiwara and S. Bornstein (Maine Medical Center Research Institute) for the assistance with $\mu \mathrm{CT}$. We also thank K. Tachikawa (Osaka Women's and Children's Hospital) for critical discussions. The authors are thankful to M. Murakami (University of California, Irvine) for pSG-Clock-myc and PER1Luc vectors. This work was supported by Grants-in-Aid for Scientific Research from the Ministry of Education, Science and Culture, Japan to MK and the JSPE Future Grant to MK.

Address correspondence to: Masanobu Kawai, Department of Bone and Mineral Research, Research Institute, Osaka Women's and Children's Hospital, 840 Murodo-cho, Izumi, Osaka 594-1101, Japan. Phone: 81.725.56.1220; E-mail: kawaim@wch.opho.jp. 
1. Ralph MR, Foster RG, Davis FC, Menaker M. Transplanted suprachiasmatic nucleus determines circadian period. Science. 1990;247(4945):975-978.

2. Green CB, Takahashi JS, Bass J. The meter of metabolism. Cell. 2008;134(5):728-742.

3. Turek FW, et al. Obesity and metabolic syndrome in circadian Clock mutant mice. Science. 2005;308(5724):1043-1045.

4. Rudic RD, et al. BMAL1 and CLOCK, two essential components of the circadian clock, are involved in glucose homeostasis. PLoS Biol. 2004;2(11):e377.

5. Vollmers C, Gill S, DiTacchio L, Pulivarthy SR, Le HD, Panda S. Time of feeding and the intrinsic circadian clock drive rhythms in hepatic gene expression. Proc Natl Acad Sci USA. 2009;106(50):21453-21458.

6. Hoogerwerf WA, et al. Clock gene expression in the murine gastrointestinal tract: endogenous rhythmicity and effects of a feeding regimen. Gastroenterology. 2007;133(4):1250-1260.

7. Sládek M, et al. Insight into the circadian clock within rat colonic epithelial cells. Gastroenterology. 2007;133(4):1240-1249.

8. Hussain MM. Regulation of intestinal lipid absorption by clock genes. Annu Rev Nutr. 2014;34:357-375.

9. Hussain MM, Pan X. Circadian regulators of intestinal lipid absorption. J Lipid Res. 2015;56(4):761-770.

10. Mukherji A, Kobiita A, Ye T, Chambon P. Homeostasis in intestinal epithelium is orchestrated by the circadian clock and microbiota cues transduced by TLRs. Cell. 2013;153(4):812-827.

11. Schachter D, Dowdle EB, Schenker H. Active transport of calcium by the small intestine of the rat. Am J Physiol. 1960;198:263-268.

12. Wróbel J. Effect of dietary calcium and photoperiod on the diurnal rhythm of active calcium transport in rat intestine. Acta Biochim Pol. 1980;27(3-4):249-255.

13. Jubiz W, Canterbury JM, Reiss E, Tyler FH. Circadian rhythm in serum parathyroid hormone concentration in human subjects: correlation with serum calcium, phosphate, albumin, and growth hormone levels. J Clin Invest. 1972;51(8):2040-2046.

14. Takarada T, et al. Bone resorption is regulated by circadian clock in osteoblasts. J Bone Miner Res. 2017;32(4):872-881.

15. Fu L, Patel MS, Bradley A, Wagner EF, Karsenty G. The molecular clock mediates leptin-regulated bone formation. Cell. 2005;122(5):803-815.

16. Xu C, et al. Circadian clock regulates bone resorption in mice. J Bone Miner Res. 2016;31(7):1344-1355.

17. Dudek M, et al. The chondrocyte clock gene Bmal1 controls cartilage homeostasis and integrity. J Clin Invest. 2016;126(1):365-376.

18. Schachter D, Rosen SM. Active transport of Ca45 by the small intestine and its dependence on vitamin D. Am J Physiol. 1959;196(2):357-362.

19. Van Cromphaut SJ, et al. Duodenal calcium absorption in vitamin D receptor-knockout mice: functional and molecular aspects Proc Natl Acad Sci USA. 2001;98(23):13324-13329.

20. Lieben $\mathrm{L}$, et al. Normocalcemia is maintained in mice under conditions of calcium malabsorption by vitamin D-induced inhibition of bone mineralization. J Clin Invest. 2012;122(5):1803-1815.

21. Yang X, et al. Nuclear receptor expression links the circadian clock to metabolism. Cell. 2006;126(4):801-810

22. Van Cromphaut SJ, et al. Intestinal calcium transporter genes are upregulated by estrogens and the reproductive cycle through vitamin D receptor-independent mechanisms. J Bone Miner Res. 2003;18(10):1725-1736.

23. Zella LA, Kim S, Shevde NK, Pike JW. Enhancers located within two introns of the vitamin D receptor gene mediate transcriptional autoregulation by 1,25-dihydroxyvitamin D3. Mol Endocrinol. 2006;20(6):1231-1247.

24. Doi M, Hirayama J, Sassone-Corsi P. Circadian regulator CLOCK is a histone acetyltransferase. Cell. 2006;125(3):497-508.

25. Srivastava AK, Bhattacharyya S, Li X, Mohan S, Baylink DJ. Circadian and longitudinal variation of serum C-telopeptide, osteocalcin, and skeletal alkaline phosphatase in C3H/HeJ mice. Bone. 2001;29(4):361-367.

26. Kopic S, Geibel JP. Gastric acid, calcium absorption, and their impact on bone health. Physiol Rev. 2013;93(1):189-268.

27. Kondratov RV, Chernov MV, Kondratova AA, Gorbacheva VY, Gudkov AV, Antoch MP. BMAL1-dependent circadian oscillation of nuclear CLOCK: posttranslational events induced by dimerization of transcriptional activators of the mammalian clock system. Genes Dev. 2003;17(15):1921-1932.

28. Kwon I, et al. BMAL1 shuttling controls transactivation and degradation of the CLOCK/BMAL1 heterodimer. Mol Cell Biol. 2006;26(19):7318-7330.

29. Spengler ML, et al. Core circadian protein CLOCK is a positive regulator of NF-кB-mediated transcription. Proc Natl Acad Sci USA. 2012;109(37):E2457-E2465.

30. Tessarz P, Kouzarides T. Histone core modifications regulating nucleosome structure and dynamics. Nat Rev Mol Cell Biol. 2014;15(11):703-708.

31. Hirayama J, et al. CLOCK-mediated acetylation of BMAL1 controls circadian function. Nature. 2007;450(7172):1086-1090.

32. Nader N, Chrousos GP, Kino T. Circadian rhythm transcription factor CLOCK regulates the transcriptional activity of the glucocorticoid receptor by acetylating its hinge region lysine cluster: potential physiological implications. FASEB J. 2009;23(5):1572-1583

33. Takeda S, et al. Leptin regulates bone formation via the sympathetic nervous system. Cell. 2002;111(3):305-317.

34. Williams EK, Chang RB, Strochlic DE, Umans BD, Lowell BB, Liberles SD. Sensory neurons that detect stretch and nutrients in the digestive system. Cell. 2016;166(1):209-221.

35. Bohórquez DV, Liddle RA. The gut connectome: making sense of what you eat. J Clin Invest. 2015;125(3):888-890.

36. Bonaz B, Bazin T, Pellissier S. The vagus nerve at the interface of the microbiota-gut-brain axis. Front Neurosci. 2018;12:49.

37. Holmbäck U, et al. Endocrine responses to nocturnal eating--possible implications for night work. Eur J Nutr. 2003;42(2):75-83.

38. Shimba S, et al. Deficient of a clock gene, brain and muscle Arnt-like protein-1 (BMAL1), induces dyslipidemia and ectopic fat formation. PLoS ONE. 2011;6(9):e25231.

39. Yadav VK, et al. Lrp5 controls bone formation by inhibiting serotonin synthesis in the duodenum. Cell. 2008;135(5):825-837.

40. Benn BS, et al. Active intestinal calcium transport in the absence of transient receptor potential vanilloid type 6 and calbindin-D9k. Endocrinology. 2008;149(6):3196-3205.

41. Mordes JP, el Lozy M, Herrera MG, Silen W. Effects of vagotomy with and without pyloroplasty on weight and food intake in rats. Am J Physiol. 1979;236(1):R61-R66.

42. Lorenz DN, Goldman SA. Vagal mediation of the cholecystokinin satiety effect in rats. Physiol Behav. 1982;29(4):599-604.

43. DeMambro VE, et al. Gender-specific changes in bone turnover and skeletal architecture in igfbp-2-null mice. Endocrinology. 
2008;149(5):2051-2061.

44. Beamer WG, et al. Genetic dissection of mouse distal chromosome 1 reveals three linked BMD QTLs with sex-dependent regulation of bone phenotypes. J Bone Miner Res. 2007;22(8):1187-1196.

45. Frost HM. Preparation of thin undecalcified bone sections by rapid manual method. Stain Technol. 1958;33(6):273-277.

46. Parfitt AM, et al. Bone histomorphometry: standardization of nomenclature, symbols, and units. Report of the ASBMR Histomorphometry Nomenclature Committee. J Bone Miner Res. 1987;2(6):595-610. 